\title{
HYPERURICEMIA INDUCED BY SOME ANTIHYPERTENSIVES AND URICOSURIC DRUGS IN OXONATE-TREATED RATS
}

\author{
Yukio YONETANI, Mitsuo ISHII and Kazumi IWAKI \\ Shionogi Research Laboratories, Shionogi \& Co., Ltd. \\ Fukushima-ku, Osaka 553, Japan
}

Accepted June 20, 1980

\begin{abstract}
Effects of antihypertensive and uricosuric drugs were studied on the plasma and urinary levels of uric acid in oxonate-treated rats. We made use of animals with a catheterized aorta to successively collect blood samples and this procedure simplified the evaluation of progressive changes of plasma uric acid, under successive loading with potassium oxonate. The plasma uric acid level of the oxonate-treated rats was increased even with a single administration of diuretic chlorothiazides, furosemide, diazoxide and also uricosuric drugs such as tienilic acid and probenecid. On the other hand, a wellmaintained plasma uric acid level was also produced by exogenously administered uric acid in rats which had been given allopurinol and potassium oxonate. Diazoxide, tienilic acid and probenecid increased the plasma uric acid, while diuretic chlorothiazides did not. Furosemide tended to decrease the plasma uric acid level at the early stage of administration to rats treated with allopurinol, oxonate and uric acid, but increased these levels several hours later when the effect was studied by uric acid loading test with rats treated with allopurinol and oxonate. These effects also appeared as changes in the urine-excreted uric acid. Thus, the oxonate-treated rats demonstrated an acutely induced hyperuricemia not only with certain antihypertensives, but also with uricosuric drugs. The utility of these procedures for evaluating the hyperuricemic and uricosuric effects of drugs is discussed.
\end{abstract}

Since selective inhibitors of urate oxidase were reported by Fridovich (1) and Iwata et al. (2), the utility of inhibitor-treated animals has enabled a better understanding of various problems related to hyperuricemia (3).

However, it has not been clarified whether such an approach is feasible for predicting the hyperuricemic or uricosuric effects of drugs; in particular, problems arising from species differences in the renal functions for uric acid excretion should be further investigated. Hyperuricemia is sometimes reported to be an undesirable side effect of some antihypertensives (4-8), and a number of agents have been practically utilized as uricosuric drugs.

However, the characterization of these drugs has been mainly developed in the clinical field, and therefore a useful model for evaluating characteristics in animal studies has long been awaited. The present study was undertaken to evaluate the utility of potassium oxonate-treated rats as models for such a purpose. Acutely induced hyperuricemia was demonstrated by some antihypertensive and uricosuric drugs.

\section{MATERIALS AND METHODS}

Animals: Nine-week-old male Wistar strain rats were maintained on a solid diet CA-1 (Japan CLEA Co., Tokyo) and tap water ad libitum. Experiments were done using rats 
with or without aortic catheterization for blood collection and intraperitoneal drug administration. The operation was performed five days before the experiment as follows: a $1 \times 10 \mathrm{~mm}$ stainless T-tube fixed at one end of polyethylene tubing (INTRAMEDIC, Clay Adams Co.) was inserted into the abdominal aorta, and the other terminal was led out at the back of the neck. Another tubing for drug administration was run from the abdomen to the same position of the neck. The tubing for blood collection was filled with $200 \mathrm{units} / \mathrm{ml}$ of heparincontaining saline, while the one for drug treatment was filled with saline. The exit terminals of both tubings were sealed and put in a capsule to prevent damage from chewing. The operated animals were then maintained in individual cages and the tubings washed every day.

Drugs: Drugs administered were potassium oxonate (Calbiochem), allopurinol (Sigma), uric acid (Tokyo Kasei), furosemide (Hoechst Japan), probenecid (Sigma), diazoxide (Schering) and others synthesized in our laboratory by Dr. H. Itazaki. The drugs were suspended in $1 \%$ gum arabic solution except for furosemide solution for injection. All drugs were administered at $2 \mathrm{ml} / \mathrm{kg}$ i.p. or p.o.

Administration of drugs and collection of blood and urine: Three types of experiments, with the timetables for drug administration and blood collection given in Fig. 1, were carried out.

The first type with catheterized animals was done with successive administration of potassium oxonate $(250 \mathrm{mg} / \mathrm{kg}$ i.p.) every two hours. The second type was performed with rats treated with allopurinol, oxonate and uric acid. Potassium oxonate $(250 \mathrm{mg} / \mathrm{kg}$ i.p.)

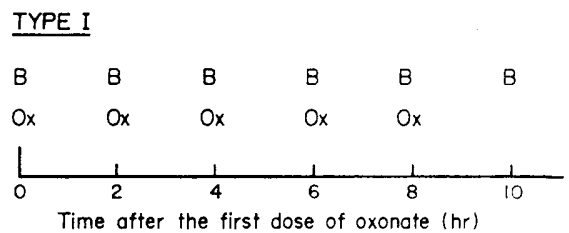

\section{TYPE II}

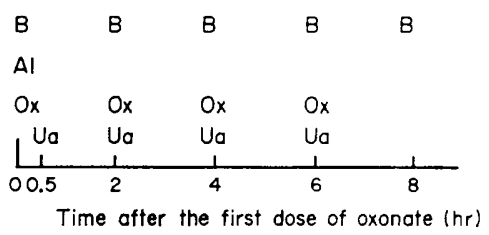

TYPE III

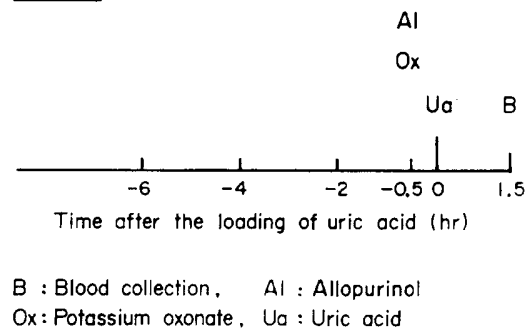

FIG. 1. Timelables for drug administration and blood collection. 
was also administered every two hours, allopurinol $(100 \mathrm{mg} / \mathrm{kg}$ p.o. $)$ was given only once concomitantly with the first dose of oxonate, and then uric acid was administered $50 \mathrm{mg} / \mathrm{kg}$ i.p. at $30 \mathrm{~min}$ after the first dose of oxonate and $25 \mathrm{mg} / \mathrm{kg}$ i.p. concomitantly with the successive doses of oxonate. The arterial blood from the catheter was collected at the indicated times prior to the administration of drugs. The third type of experiment was carried out using rats not subjected to catheterization.

Allopurinol (100 mg/kg p.o.) and potassium oxonate $(250 \mathrm{mg} / \mathrm{kg}$ i.p.) were administered, then uric acid was intraperitoneally loaded $30 \mathrm{~min}$ later. Blood was collected by decapitation at $1.5 \mathrm{hr}$ after the loading of uric acid. In the second type of experiment, urine was collected using individual metabolism cages. The duration for urine collection is described with the experimental results.

Determination of uric acid and allantoin: Blood samples were cooled in ice water immediately after collection, then the plasma was separated as soon as possible. Plasma uric acid and allantoin were estimated basically according to the methods of Sumi et al. $(9,10)$. Uric acid was determined with a concentration of urate oxidase much higher than that in the original procedure in order to nullify the influence of urate oxidase inhibitor included in the samples. Allantoin was estimated by determining the reduced amounts of NADH during the glyoxylate reductase reaction. In the procedure, $0.5 \mathrm{ml}$ of the deproteinized sample solution was mixed with $0.5 \mathrm{ml}$ of distilled water and $0.1 \mathrm{ml}$ of $1.0 \mathrm{~N}$ sodium hydroxide solution. The mixture was heated for $7 \mathrm{~min}$ in a boiling water bath, then rapidly cooled in ice water. After addition of $0.1 \mathrm{ml}$ of $1.5 \mathrm{~N}$ hydrochloric acid, the mixture was heated again for $5 \mathrm{~min}$ in a boiling water bath, then cooled in ice water. Next, $1 \mathrm{ml}$ of $0.25 \mathrm{M}$ phosphate buffer, $\mathrm{pH} 6.4$ and $0.2 \mathrm{ml}$ of NADH solution (4 mg of NADH was dissolved in $50 \mathrm{ml}$ of $0.001 \mathrm{M}$ Tris-hydrochloric acid buffer, $\mathrm{pH} 9.0$ ) were added to the mixture. The reduced amounts of NADH in the reaction mixture during the incubation for $60 \mathrm{~min}$ at room temperature with $0.02 \mathrm{ml}$ of glyoxylate reductase solution $(0.1 \mathrm{ml}$ of spinach leaf enzyme, $5 \mathrm{mg}$ protein/ml preparation, Boehringer Mannheim Co., was diluted with $0.4 \mathrm{ml}$ of $0.01 \mathrm{M}$ phosphate buffer, pH 7.0, containing $0.005 \mathrm{M}$ ethylenediamine-tetraacetic acid) were fluorometrically estimated at the wavelength of $460 \mathrm{~m} \mu$ with the excitation at $365 \mathrm{~m} \mu$. The reduction of $\mathrm{NADH}$ during the enzyme reaction was mainly dependent upon the glyoxylic acid produced from allantoin, but a false reaction due to the high concentration of uric acid could not be ruled out. Accordingly, calibration curves for estimating the reduced amounts of NADH were made for both uric acid and allantoin, then the allantoin concentration in the samples was calculated after eliminating the effect of uric acid in the assay. The concentrations of urinary uric acid and allantoin were determined by the same procedure used for these assessments in the plasma.

\section{RESULTS}

First, rats with a prolonged high level of plasma uric acid were produced by administration of potassium oxonate. According to Johnson et al. (11), $250 \mathrm{mg} / \mathrm{kg}$ i.p. was sufficient to cause an effective inhibition of urate oxidase in rats. As shown in Fig. 2-A, the successive 


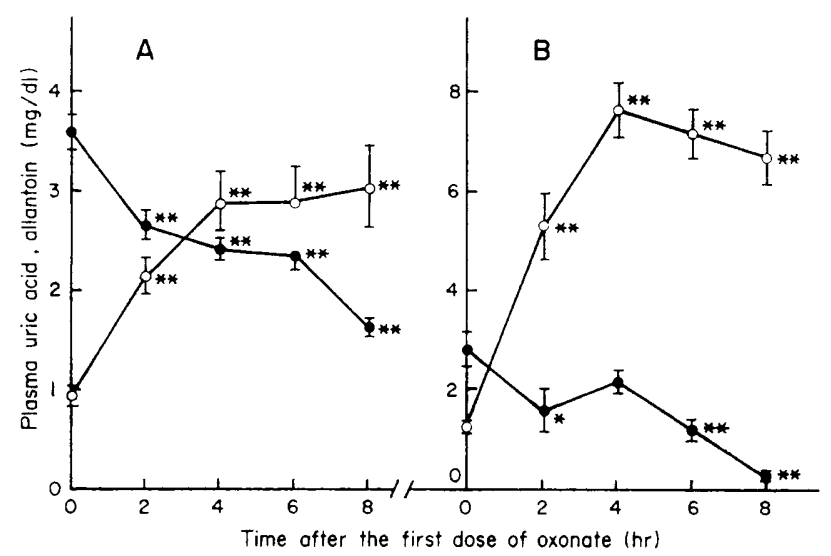

FIG. 2. Changes of plasma uric acid and allantoin in rats treated with oxonate (A) or allopurinol, oxonate and uric acid (B). Procedures for drug administration and blood collection are described in the text. The open circles show the mean uric acid value of 4 or 5 animals, and the filled ones are those for allantoin. Bars give the standard errors. *, ${ }^{* *}$ : Significantly different from the initial level at $\mathrm{p}<0.05$ and 0.01 , respectively.

administration of potassium oxonate every two hours produced a high level of uric acid and a decrease of allantoin concentration. Such a prolonged high level of plasma uric acid could be also produced by exogenously administered uric acid in animals which had been given allopurinol and potassium oxonate (Fig. 2-B).

These two models were used to evaluate the hyperuricemic effects of some antihypertensives and to characterize uricosuric drugs. The concentration of plasma uric acid in these models was readily affected by diverse drugs, but the allantoin level remained unchanged. Thus, only the changes of the plasma uric acid are described below.

Various antihypertensives readily produced an increase of the plasma uric acid level in oxonate-treated rats when administered only once, concomitantly, with the first dose of oxonate. As demonstrated in Fig. 3, diuretic chlorothiazides such as hydrochlorothiazide, trichloromethiazide and cyclopenthiazide gradually increased the plasma uric acid during the successive administration of oxonate.

However, as demonstrated with hydrochlorothiazide, these diuretic chlorothiazides tended to decrease the plasma uric acid level at the early stage upon administration of higher doses. Cyclopenthiazide had the most potent effect among the tested chlorothiazides, and $20 \mathrm{mg} / \mathrm{kg}$ produced a more apparent increase in the plasma uric acid than that seen with $2 \mathrm{mg} / \mathrm{kg}$. On the other hand, diazoxide increased the plasma uric acid only at the early stage, and furosemide produced a rapid and prolonged hyperuricemia in response to the doses. An acutely induced hyperuricemia in oxonate-treated rats was also seen with uricosuric drugs such as tienilic acid and probenecid (Fig. 4).

Next, studies were carried out with rats treated with allopurinol, oxonate and uric acid to determine whether the hyperuricemic effects of antihypertensives and uricosuric drugs in oxonate-treated animals were due to their effects on renal excretion of uric acid. The test 


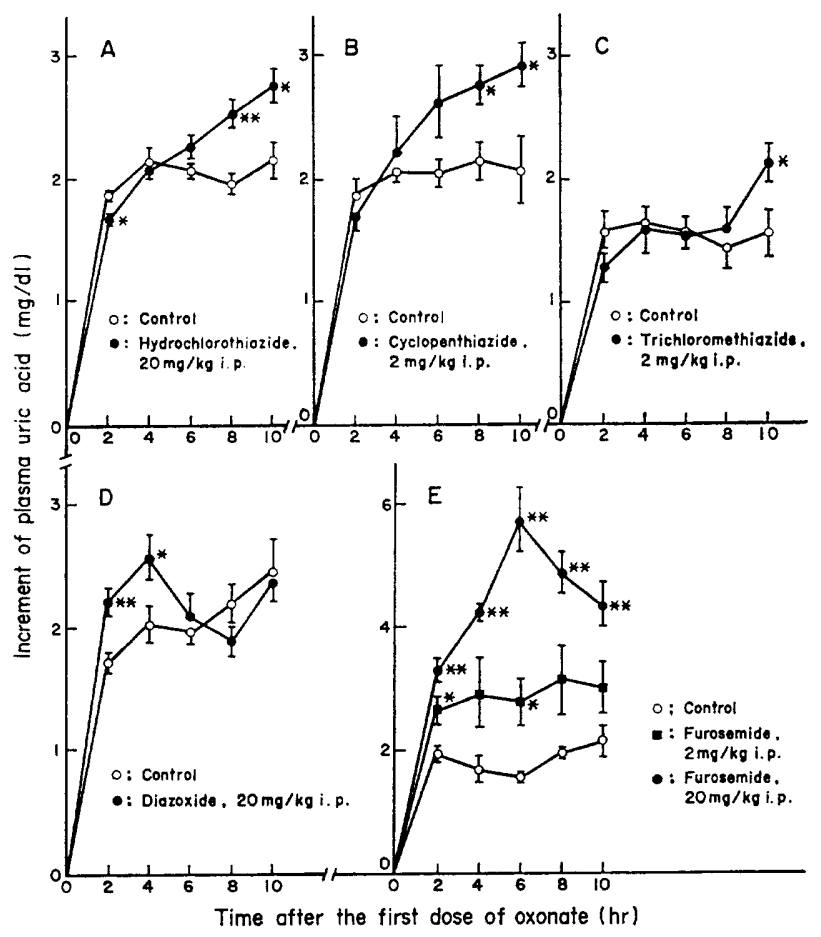

FIG. 3. Effects of antihypertensives on plasma uric acid in oxonate-treated rats. Test drugs were administered only once concomitantly with the first dose of oxonate. Values represent means \pm standard errors of 6 or 7 animals. *, **: Significantly different from the control group at $\mathrm{p}<0.05$ and 0.01 , respectively.

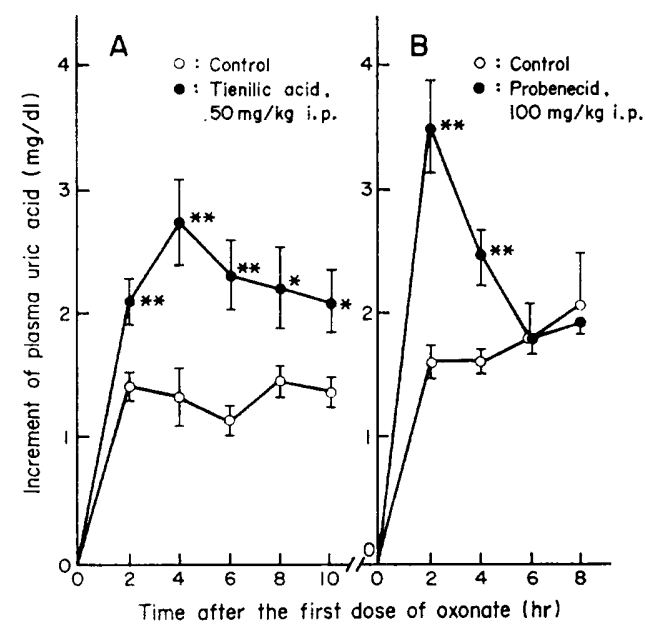

Fig. 4. Effects of uricosuric drugs on plasma uric acid in oxonate-treated rats. Test drugs were administered only once concomitantly with the first dose of oxonate. Values represent means \pm standard errors of 5 or 6 animals. *, **: Significantly different from the control group at $\mathrm{p}<0.05$ and 0.01 , respectively. 
drugs were first administered concomitantly with the first dose of oxonate, as in the case of oxonate-treated rats. As seen in Fig. 5-A, cyclopenthiazide and furosemide did not increase plasma uric acid levels and furosemide decreased it at the early stage. On the other hand, tienilic acid and probenecid produced a hyperuricemic effect (Fig. 5-B), as did diazoxide (Fig. 5-C).

As shown in Table 1, the treatment with oxonate or oxonate and uric acid was diuretic and hyperuricosuric. In particular, the diuretic effect in the rats treated with allopurinol, oxonate and uric acid was progressively enhanced, and all the test drugs were without effect on plasma uric acid levels when concomitantly given with the second administration of

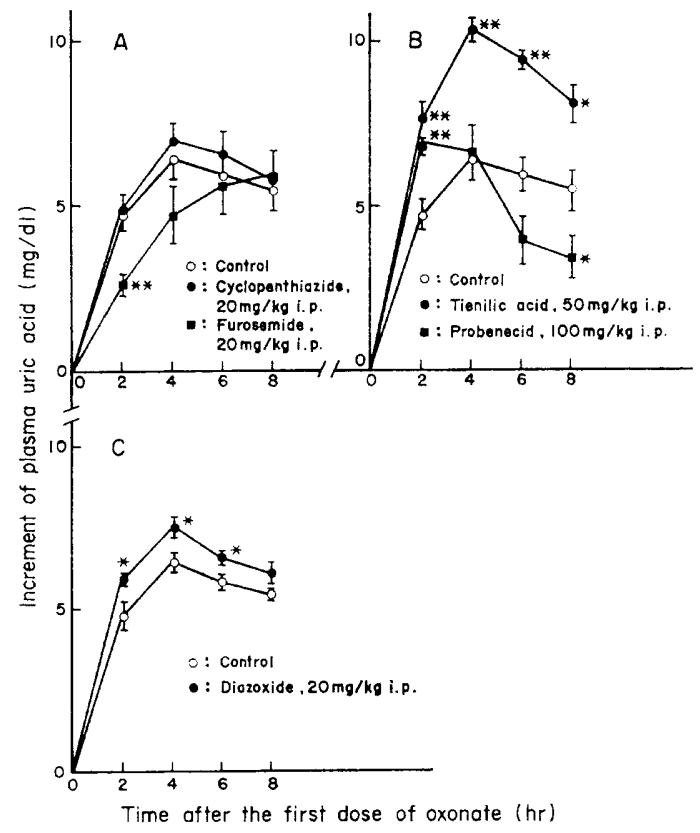

FIG. 5. Effects of diuretic antihypertensives, uricosuric drugs and diazoxide on plasma uric acid in rats treated with allopurinol, oxonate and uric acid. Test drugs were administered only once concomitantly with the first dose of oxonate. Values represent means \pm standard errors of 5 or 7 animals. *, **: Significantly different from the control group at $\mathrm{p}<0.05$ and 0.01 , respectively.

TABLE 1. Diuretic and hyperuricosuric effects of treatment with oxonate and with allopurinol, oxonate and uric acid

\begin{tabular}{lccc}
\hline Treatment & $\mathrm{n}$ & $\begin{array}{c}\text { Urine volume } \\
(\mathrm{ml})\end{array}$ & $\begin{array}{c}\text { Urinary uric acid } \\
(\mathrm{mg})\end{array}$ \\
\hline Control & 6 & $1.2 \pm 0.1$ & $0.40 \pm 0.06$ \\
Oxonate & 7 & $6.0 \pm 0.3^{* *}$ & $7.98 \pm 0.49^{* *}$ \\
Allopurinol, oxonate, uric acid & 6 & $12.0 \pm 1.0^{* *}$ & $19.94 \pm 1.67^{* *}$ \\
\hline
\end{tabular}

Methods for drug administration are described in the text. Urine was collected for $6 \mathrm{hr}$ after the second dose of oxonate. Data represent the mean \pm standard error.

**: Significantly different from the control level at $\mathrm{p}<0.01$. 
oxonate.

The time necessary for inhibition of uric acid production and metabolism with administration of allopurinol and potassium oxonate was determined. This was done by administering catecholamine which rapidly stimulated the production of uric acid, as reported previously (12). The stimulation affected the plasma level of uric acid and allantoin, when given within $30 \mathrm{~min}$ after the administration of allopurinol and oxonate (data not included).

However, as demonstrated in Fig. 6, diazoxide, tienilic acid and probenecid produced an increase of the plasma uric acid when administered $30 \mathrm{~min}$ after allopurinol, in rats treated with allopurinol, oxonate and uric acid.

Table 2 shows the changes in urine-excreted uric acid in rats for which changes in the

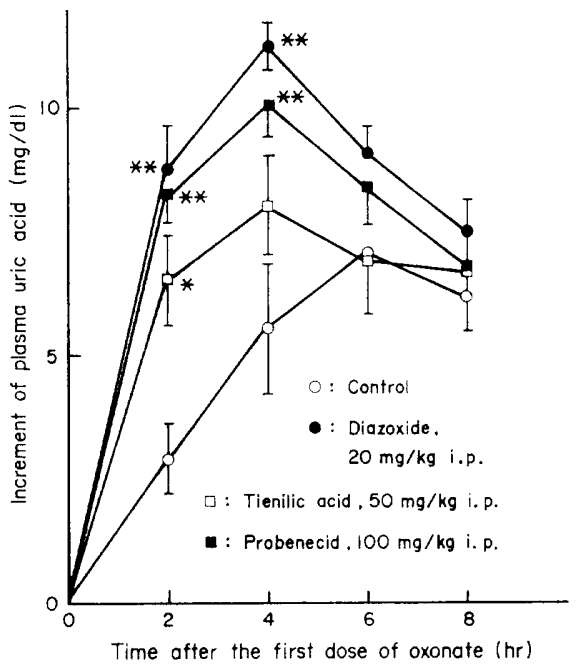

FIG. 6. Effects of diazoxide, tienilic acid and probenecid on plasma uric acid in rats treated with allopurinol, oxonate and uric acid. Test drugs were administered $30 \mathrm{~min}$ after the treatment with allopurinol. Values represent means \pm standard errors of 4 or 6 animals. *, ${ }^{* *}$ : Significantly different from the control group at $\mathrm{p}<0.05$ and 0.01 , respectively.

TABLE 2. Effects of diuretic antihypertensives and uricosuric drugs on urinary excretion of uric acid in rats treated with allopurinol, oxonate and uric acid

\begin{tabular}{|c|c|c|c|}
\hline \multirow{2}{*}{ Treatment } & \multirow{2}{*}{$n$} & \multicolumn{2}{|c|}{ Urinary uric acid (mg) } \\
\hline & & First period & Second period \\
\hline Control & 5 & $1.46+0.43$ & $15.77=1.16$ \\
\hline Cyclopenthiazide & 6 & $1.17-0.15$ & $14.82-0.51$ \\
\hline Furosemide & 6 & $5.15 \pm 0.34^{* *}$ & $9.76+0.84^{* *}$ \\
\hline Tienilic acid & 6 & $0.48-0.06^{*}$ & $15.26 \pm 1.27$ \\
\hline Probenecid & 7 & $1.08+0.26$ & $16.67 \div 1.45$ \\
\hline
\end{tabular}

The first period for urine collection was for $2 \mathrm{hr}$ after administration of the test drugs, while the second period was for the subsequent $6 \mathrm{hr}$. Methods for drug administration are described in Fig. 5 and the text. Data represent the mean : standard error. *, ${ }^{* *}$ : Significantly different from the control level at $\mathrm{p}<0.05$ and 0.01 , respectively. 
plasma uric acid are shown in Fig. 5-A and -B. Cyclopenthiazide had no effect, even on the amounts of urine-excreted uric acid, while furosemide markedly increased the excretion of uric acid at the early stage, then decreased it later. Tienilic acid decreased the excretion of uric acid at the early stage, but such an effect with probenecid was not appreciable.

Diazoxide was studied in another series of experiments, and its effect on urine-excreted uric acid was the same as that of probenecid.

However, as shown in Table 3, diazoxide and probenecid decreased the excretion of uric acid at the early stage in another experiment, whereas the effect of tienilic acid could not be detected. In this experiment, urine collection at the early stage was performed for a longer period than in the previous experiment in order to better evaluate the effects of diazoxide and probenecid, and the changes of plasma uric acid are shown in Fig. 6.

TABLE 3. Effects of diazoxide, tienilic acid and probenecid on urinary excretion of uric acid in rats treated with allopurinol, oxonate and uric acid

\begin{tabular}{lllc}
\hline Treatment & $\mathrm{n}$ & \multicolumn{2}{c}{ Urinary uric acid $(\mathrm{mg})$} \\
& & First period & Second period \\
\hline Control & 4 & $7.67 \pm 0.25$ & $13.51 \pm 0.64$ \\
Diazoxide & 6 & $2.79 \pm 0.09^{* *}$ & $13.43 \pm 1.12$ \\
Tienilic acid & 5 & $6.10 \pm 0.92$ & $13.02 \pm 1.16$ \\
Probenecid & 6 & $4.77 \pm 0.73^{*}$ & $11.93 \pm 0.59$ \\
\hline
\end{tabular}

The first period for urine collection was for $3.5 \mathrm{hr}$ after administration of the test drug, and the second one was for the subsequent $4 \mathrm{hr}$. Other experimental conditions are given in Fig. 6 and the text. Data represent the mean \pm standard error. ${ }^{*},{ }^{* *}$ : Significantly different from the control level at $\mathrm{p}<0.05$ and 0.01 , respectively.

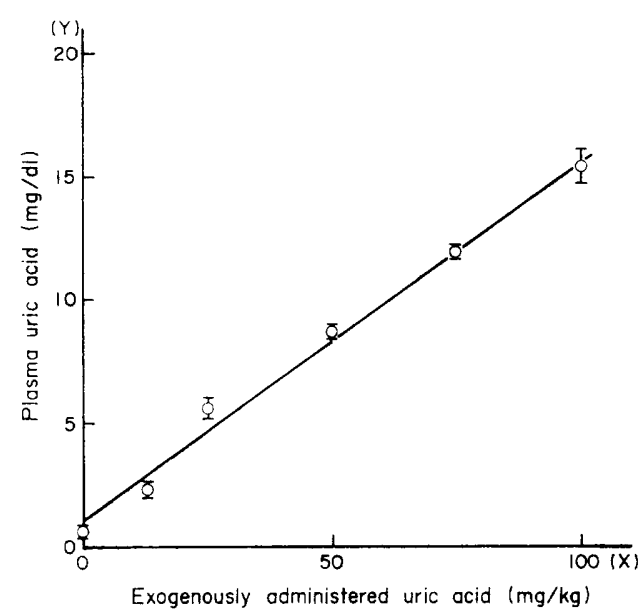

Fig. 7. Relationship between plasma uric acid level and doses of exogenously administered uric acid in rats treated with allopurinol and potassium oxonate. The experiment was carried out according to the method described in the text (the third type of experiment). Values represent means \pm standard errors of 7 or 8 animals. The calculated relationship is: $\mathrm{Y}=0.146 \mathrm{X}+1.06(\mathrm{~S} . \mathrm{D} .=1.12, \mathrm{n}=46$ ). 
Next, studies were done using rats not prepared for blood collection and drug administration. As shown in Fig. 7, exogenously administered uric acid produced a dose-responsive elevation of plasma uric acid in rats which had been given allopurinol and potassium oxonate 30 min previously.

This uric acid loading enabled assessments of effects of drugs on the renal excretion of uric acid. Diuretic antihypertensives such as cyclopenthiazide and furosemide reduced the plasma uric acid level at the early stage, as shown in Table 4. Furosemide, however, increased these levels later, while cyclopenthiazide had no such adverse effect. On the other hand, diazoxide, tienilic acid and probenecid produced a hyperuricemic effect, even in the uric acid loading test, when given concomitantly with exogenously administered uric acid (Table 5).

TABLE 4. Effects of cyclopenthazide and furosemide in the uric acid loading test with rats treated with allopurinol and oxonate

\begin{tabular}{lccc}
\hline \multicolumn{1}{c}{ Test drug } & $\begin{array}{c}\text { Time of drug } \\
\text { administration }(\mathrm{hr})\end{array}$ & $\mathrm{n}$ & $\begin{array}{c}\text { Plasma uric acid } \\
(\mathrm{mg} / \mathrm{dl})\end{array}$ \\
\hline None (Control) & & 8 & $5.85 \pm 0.47$ \\
Cyclopenthiazide & 0 & 7 & $4.27 \pm 0.34^{*}$ \\
$(20 \mathrm{mg} / \mathrm{kg})$ & -2 & 7 & $3.10 \pm 0.42^{* *}$ \\
& -4 & 7 & $4.10 \pm 0.59^{*}$ \\
& -6 & 8 & $5.33 \pm 0.51$ \\
\hline None (Control) & & 8 & $5.60 \pm 0.38$ \\
Furosemide & 0 & 7 & $3.17 \pm 0.20^{* *}$ \\
$(20 \mathrm{mg} / \mathrm{kg})$ & -2 & 7 & $7.62 \pm 0.65^{*}$ \\
& -4 & 7 & $8.38 \pm 0.16^{* *}$ \\
& -6 & 7 & $8.75 \pm 0.83^{* *}$ \\
\hline
\end{tabular}

Test drugs were given at the indicated time after the loading of uric acid $(25 \mathrm{mg} / \mathrm{kg}$ i.p.), according to the method described in the text. Data represent the mean \pm standard error. ${ }^{*},{ }^{* *}$ : Significantly different from the control level at $\mathrm{p}<0.05$ and 0.01 , respectively.

TABle 5. Effects of uricosuric drugs and diazoxide in uric acid loading test with rats treated with allopurinol and oxonate

\begin{tabular}{rlc}
\hline \multicolumn{1}{c}{ Test drug } & $\mathrm{n}$ & Plasma uric acid (mg/dl) \\
\hline None (Control) & 8 & $5.63 \pm 0.39$ \\
Probenecid, $25 \mathrm{mg} / \mathrm{kg}$ i.p. & 6 & $6.97 \pm 0.25^{*}$ \\
$50 \mathrm{mg} / \mathrm{kg}$ i.p. & 7 & $7.19 \pm 0.35^{*}$ \\
$100 \mathrm{mg} / \mathrm{kg}$ i.p. & 8 & $7.35 \pm 0.35^{* *}$ \\
$200 \mathrm{mg} / \mathrm{kg}$ p.o. & 7 & $7.11 \pm 0.36^{*}$ \\
Tienilic acid, $50 \mathrm{mg} / \mathrm{kg}$ i.p. & 7 & $7.29 \pm 0.30^{* *}$ \\
Diazoxide, $20 \mathrm{mg} / \mathrm{kg}$ i.p. & 7 & $8.57 \pm 0.33^{* *}$ \\
\hline
\end{tabular}

Test drugs were administered concomitantly with the loading of uric acid $(25 \mathrm{mg} / \mathrm{kg}$ i.p.), according to the method described in the text. Data represent the mean \pm standard error. *, **: Significantly different from the control level at $\mathrm{p}<0.05$ and 0.01 , respectively. 


\section{DISCUSSION}

Most experimental animals differ from primates not only in the metabolic process of uric acid, but also in its renal excretion (13). Thus, careful consideration should be given the utility of animals treated with urate oxidase inhibitor when evaluating the hyperuricemic effects of diverse drugs and characterizing uricosuric drugs. Smith et al. (14) reported acutely induced hyperuricemia in oxonate-treated rats given antihypertensive drugs, and their findings in the case of diazoxide and furosemide were similar to ours in the present study. However, progressive changes in plasma uric acid levels in rats on furosemide were demonstrated for the first time by the methods described in the present paper, and evaluations of the hyperuricemic effects of test drugs at lesser doses were made feasible.

Since Demartini (15) demonstrated that diuretic chlorothiazides induce hyperuricemia due to a specific inhibition of renal uric acid excretion, this explanation has generally been accepted regarding the mechanism of antihypertensive-induced hyperuricemia. Our present study on diuretic antihypertensives showed an acutely induced hyperuricemia in oxonatetreated rats, however, an adequate explanation for the renal effects was not forthcoming. Furosemide decreased the urinary excretion of uric acid several hours after the administration to rats treated with allopurinol, oxonate and uric acid (Table 2), and produced a hyperuricemia in the uric acid loading test with rats treated with allopurinol and oxonate (Table 4). These effects support its renal effect as a cause of the hyperuricemia.

On the other hand, diuretic chlorothiazides did not increase the plasma uric acid in rats treated with allopurinol, oxonate and uric acid and furosemide decreased the plasma uric acid at the early stage following treatment with allopurinol, oxonate and uric acid. These results indicate that stimulation of uric acid production as a cause of acutely induced hyperuricemia by diuretic antihypertensives in oxonate-treated animals cannot be ruled out. Thus, some diuretic antihypertensives of which hyperuricemic effects in clinical use have been well documented, demonstrated an acute hyperuricemia in oxonate-treated rats, but the causes were not always related to the inhibition of renal uric acid excretion.

Diazoxide is a nondiuretic antihypertensive drug, inhibits renal uric acid excretion (16) and produces a hyperuricemic effect (14). Its effect in rats treated with allopurinol, oxonate and uric acid well supported these characteristics. On the other hand, our results with tienilic acid and probenecid suggested that oxonate-treated rats were not suitable for evaluating the uricosuric effects of drugs dependent upon their inhibition of renal tubular reabsorption. The so-called uricosuric drugs sometimes produced a paradoxical effect on the plasma uric acid level, as the result of different doses or species characteristics with regard to renal function for uric acid excretion (13). In our experiments with oxonatetreated rats, the effects of tienilic acid and probenecid in decreasing the plasma uric acid were not related to the doses used and the hyperuricemic effects in rats treated with allopurinol, oxonate and uric acid support the idea that renal tubular secretion of uric acid predominates over the reabsorption function in oxonate-treated rats.

We determined the effect of probenecid on the plasma concentration of oxonate in oxonate-treated rats under the same experimental conditions as in Fig. 4, and found that 
probenecid increased the plasma level of oxonate at the early stage. Accordingly, the inhibited excretion of oxonate cannot be ruled out as a cause of the uricosuric drug-induced hyperuricemia in oxonate-treated animals. On the other hand, considering the absence of a suitable procedure for the characterization of uricosuric drugs in animal studies, the paradoxical effect in rats treated with allopurinol, oxonate and uric acid may be utilized to screen the drugs. The uric acid loading test in rats treated with allopurinol and oxonate was tested for such a purpose. Both uricosuric drugs and drugs inhibiting renal uric acid excretion such as diazoxide were hyperuricemic in the test.

Thus, oxonate-treated rats are not always satisfactory models for evaluating drug effects and hence should be utilized after due consideration of their characteristics of renal uric acid excretion.

Acknowledgements: We thank Dr. H. Itazaki, Shionogi Research Laboratories for synthesizing the test drugs and Dr. M. Ueda, Shionogi Research Laboratories for pertinent discussion.

\section{REFERENCES}

1) Fridovich, I.: The competitive inhibition of uricase by oxonate and by related derivatives of s-triazines. J. Biol. Chem. 240, 2491-2494 (1965)

2) Imata, H., Yamamoto, I., Gohda, E., Morita, K., Nakamura, M. and Sumi, K.: Potent competitive uricase inhibitors 2,8-diazahypoxanthine and related compounds. Biochem. Pharmacol. 22, 2237-2245 (1973)

3) Stavric, B. And Nera, E.A.: Use of the uricase-inhibited rat as an animal model in toxicology. Clin. Toxicol. 13, 47-74 (1978)

4) Laragh, J.H. and Heinemann, H.O.: Effect of chlorothiazide on electrolyte transport in man. J. Am. med. Ass. 166, 145-152 (1958)

5) Monroe, K.E., Grant, L.H., Sasahara, A.A. and Littmann, D.: Effect of chlorothiazide therapy on serum uric acid and uric acid excretion. New Engl. J. Med. 261, 290-292 (1959)

6) Dollery, G.T., Duncan, H. And Schumer, B.: Hyperuricemia related to treatment of hypertension. Brit. med. J. 2, 832-835 (1960)

7) Kelley, W.N., Grobner, W. and Holmes, E.: Current concepts in the pathogenesis of hyperuricemia. Metabolism 22, 939-959 (1973)

8) Sмттн, C.J.: Disorders associated with hyperuricemia. Arth. Rheum. 18, 713-719 (1975)

9) Sumi, T., Umeda, Y., Kishi, Y., Takahashi, K. and Kakimoto, F.: The enzymatic spectrofluorimetric determination of uric acid in microsamples of plasma by using p-hydroxyphenylacetic acid as a fluorophor. Clin. Chim. Acta. 73, 233-239 (1976)

10) Sumi, T., Umeda, Y., Kishi, Y., Kakimoto, F. and Takahashi, K.: A spectrofluorometric method for determining plasma allantoin based on the glyoxylate reductase reaction. Analyt. Biochem. 75, 563-567 (1976)

11) Johnson, W.J., Stavric, B. And Chartrand, A.: Uricase inhibition in the rat by s-triazines: an animal model for hyperuricemia and hyperuricosuria. Proc. Soc. exp. Biol. Med. 131, 8-12 (1969)

12) Yonetani, Y., Ishit, M. and Ogawa, Y.: Stimulation by catecholamine of purine catabolism in rats and chickens. Japan. J. Pharmacol. 29, 211-221 (1979)

13) Guttman, A.B.: Uricosuric drugs, with special reference to probenecid and sulfinpyrazone. Advances Pharmacol. 4, 91-142 (1966)

14) Smith, R.D., Essenburg, A.D. and Kaplan, H.R.: The oxonate pretreated rat as a model for evaluating hyperuricemic effects of antihypertensive drugs. Clin. Exptl. Hypertension. 1, 487-504 (1979) 
15) Demartini, F.E.: Hyperuricemia induced by drugs. Arth. Rheum. 8, 823-829 (1965)

16) Thompson, G.R.: The effect of diazoxide, potassium chloride, and ammonium chloride on serum and urinary uric acid. Arth. Rheum. 8, 830-835 (1965) 